\title{
SUBDIFFERENTIABILITY OF CONVEX FUNCTIONS WITH VALUES IN AN ORDERED VECTOR SPACE
}

\author{
JOCHEM ZOWE ${ }^{1}$
}

\begin{abstract}
.
It was shown by Valadier [8] that a convex function defined on a topological vector space $X$ with values in a topological order complete vector lattice $Y$ is subdifferentiable (even regularly subdifferentiable) at each point, where the function is continuous. We will prove that under some assumptions on $X$ and the order cone $C$ this even holds, if $Y$ is an ordered topological vector space. Furthermore we will see that under our assumptions on $X$ and $C$ the Gateaux differentiability of a convex function is equivalent to the existence of only one subgradient. Our result apply e.g. if $X$ is a separable reflexive Banach space and $Y$ is a semireflexive locally convex space ordered by a cone with a weakly compact base.
\end{abstract}

\section{Introduction and notations.}

Throughout the following let $X$ and $Y$ be separated locally convex vector spaces over $\mathrm{R}$ and let $Y$ be ordered by a closed convex proper cone $C$. We write $z \leqq y$ for $z, y \in Y$ if $y-z \in C$. With $X^{\prime}, Y^{\prime}$ we denote the topological duals of $X$ and $Y$ and with $\langle\cdot, \cdot\rangle$ the canonical bilinear forms on the dualities $\left\langle X, X^{\prime}\right\rangle$ and $\left\langle Y, Y^{\prime}\right\rangle$. Furthermore let $\sigma(\cdot, \cdot)$, $\tau(\cdot, \cdot), \beta(\cdot, \cdot)$ stand for the weak, Mackey- and strong topologies with respect to the dual pairs $\left\langle X, X^{\prime}\right\rangle$ and $\left\langle Y, Y^{\prime}\right\rangle$. We write for example $X^{\prime}{ }_{\sigma}$ for $X^{\prime}$ under $\sigma\left(X^{\prime}, X\right), N \sigma\left(X^{\prime}, X\right)$ for the neighbourhood filter of 0 in $X^{\prime}{ }_{\sigma}, A^{\circ}{ }_{\sigma}\left(\bar{A}_{\sigma}\right)$ for the interior (closure) of a set $A \subset X^{\prime}{ }_{\sigma}$ etc. If $A \subset X^{\prime}$ is convex then $\bar{A}_{\sigma}=\bar{A}_{\tau}$ and we omit the subscript.

We will consider a function $f$ mapping a nonvoid convex subset $K$ of $X$ into $Y$ such that for all $x_{1}, x_{2} \in K$ and $\lambda \in \mathrm{R}, 0 \leq \lambda \leq 1$,

$$
f\left(\lambda x_{1}+(1-\lambda) x_{2}\right) \leqq \lambda f\left(x_{1}\right)+(1-\lambda) f\left(x_{2}\right) .
$$

Received August 8, 1973.

1 This paper was written during the author's stay in the summer 1973 as a guest at the Department of Mathematics of the University of Dundee, Scotland. 
$f$ is called a convex function. It is assumed throughout the following without further mentioning that

(1.1) $x_{0} \in K^{\circ}$ and $f$ is continuous at $x_{0}$, when $f$ is regarded as a mapping of $X$ into $Y_{\sigma}$.

What we are interested in, is the set

$$
\partial f\left(x_{0}\right):=\left\{T \in \mathscr{L}(X, Y): T\left(x-x_{0}\right) \leqq f(x)-f\left(x_{0}\right) \text { for all } x \in K\right\}
$$

of subgradients of $f$ at $x_{0}$ (here $\mathscr{L}(X, Y)$ stands for the set of continuous linear mappings from $X$ into $Y)$. $\partial f\left(x_{0}\right)$ is called the subdifferential of $f$ at $x_{0}$. In the special case $Y=\mathrm{R}$ the subdifferential is a nonvoid convex compact subset of $X^{\prime}{ }_{\sigma}$ (see [4]). Each $y^{\prime}$ in the dual cone of $C$

$$
C^{\prime}:=\left\{y^{\prime} \in Y^{\prime}:\left\langle C, y^{\prime}\right\rangle \geq 0\right\},
$$

defines a convex functional

$$
y^{\prime} \circ f(x):=\left\langle f(x), y^{\prime}\right\rangle \quad \text { for all } x \in K .
$$

Therefore

$$
\partial\left(y^{\prime} \circ f\right)\left(x_{0}\right), y^{\prime} \in C^{\prime}, \text { is a nonvoid convex compact subset of }{X^{\prime}}_{\sigma} .
$$

(Here

$$
\left.\partial\left(y^{\prime} \circ f\right)\left(x_{0}\right)=\left\{x^{\prime} \in X^{\prime}:\left\langle x-x_{0}, x^{\prime}\right\rangle \leq y^{\prime} \circ f(x)-y^{\prime} \circ f\left(x_{0}\right) \text { for all } x \in K\right\} .\right)
$$

Valadier [8] showed that an analoguous result holds for $f$ itself, if $Y$ is an order complete vector lattice. In his proof this assumption on $Y$ is essentially used to show that $\partial f\left(x_{0}\right)$ is nonvoid. We are going to demonstrate that this result remains valid for an ordered vector space, if $\left(C^{\prime}\right)^{\circ}{ }_{\tau} \neq \varnothing$. Roughly speaking, our idea is the following: First note that the transpose $S$ of a $T \in \partial f\left(x_{0}\right)$ is a continuous linear mapping from $Y^{\prime}$ 。 into $X^{\prime}{ }_{\sigma}$ and that $S y^{\prime} \in \partial\left(y^{\prime} \circ f\right)\left(x_{0}\right)$ for all $y^{\prime} \in C^{\prime}$. Conversely, we will construct a continuous linear mapping $S$ from $Y^{\prime}{ }_{\sigma}$ into $X^{\prime}{ }_{\sigma}$ with

$$
S y^{\prime} \in \partial\left(y^{\prime} \circ f\right)\left(x_{0}\right), \quad y^{\prime} \in C^{\prime},
$$

and the transpose will belong to $2 f\left(x_{0}\right)$. The existence of a $S: C^{\prime} \rightarrow X^{\prime}$ such that $S y^{\prime} \in \partial\left(y^{\prime} \circ f\right)\left(x_{0}\right)$ is an easy consequence of (1.4). The important point is that under the assumption $\left(C^{\prime}\right)^{\circ}{ }_{\tau} \neq \varnothing$ the mapping $S$ can be chosen to be linear and continuous. 


\section{Auxiliary propositions.}

For the proof of our principal auxiliary result, proposition 2.5, we need some further information about the sets $\partial\left(y^{\prime} \circ f\right)\left(x_{0}\right), y^{\prime} \in C^{\prime}$. First let us state some simple well-known facts. The directional derivative of $y^{\prime} \circ f, y^{\prime} \in C^{\prime}$, at $x_{0}$ in the direction $h$,

$$
\left(y^{\prime} \circ f\right)^{\prime}\left(x_{0} ; h\right)=\lim \lambda^{-1}\left(y^{\prime} \circ f\left(x_{0}+\lambda h\right)-y^{\prime} \circ f\left(x_{0}\right)\right), \quad \lambda \rightarrow 0, \lambda>0,
$$

is a positively homogeneous, subadditive functional in $h$, defined for all $h \in X$. We have

$$
\left(y^{\prime} \circ f\right)^{\prime}\left(x_{0} ; h\right)=\inf \left\{\lambda^{-1}\left(y^{\prime} \circ f\left(x_{0}+\lambda h\right)-y^{\prime} \circ f\left(x_{0}\right)\right): \lambda>0, x_{0}+\lambda h \in K\right\},
$$
and thus

Proposition 2.1. Let $y^{\prime} \in C^{\prime}$. Then $x^{\prime} \in \partial\left(y^{\prime} \circ f\right)\left(x_{0}\right)$ if and only if

$$
\left\langle h, x^{\prime}\right\rangle \leq\left(y^{\prime} \circ f\right)^{\prime}\left(x_{0} ; h\right) \quad \text { for all } h \in X \text {. }
$$

Proposition 2.2. Let $y^{\prime} \in C^{\prime}$ and $h_{0} \in X$. Then

$$
\left\langle h_{0}, x_{0}{ }^{\prime}\right\rangle=\left(y^{\prime} \circ f\right)^{\prime}\left(x_{0} ; h_{0}\right)
$$

for some $x_{0}{ }^{\prime} \in \partial\left(y^{\prime} \circ f\right)\left(x_{0}\right)$.

Proof. Consider the convex functional $p(h):=f^{\prime}\left(x_{0} ; h\right)$. We have

$$
p( \pm h) \leq y^{\prime} \circ f\left(x_{0} \pm h\right)-y^{\prime} \circ f\left(x_{0}\right)
$$

for $h$ small enough such that $x_{0} \pm h \in K$, and because of $p(h)+p(-h) \geq$ $p(0)=0$ we get

$$
p(h) \leq \max \left\{\left|y^{\prime} \circ f\left(x_{0}+h\right)-y^{\prime} \circ f\left(x_{0}\right)\right|,\left|y^{\prime} \circ f\left(x_{0}\right)-y^{\prime} \circ f\left(x_{0}-h\right)\right|\right\} .
$$

Since $y^{\prime} \circ$ is continuous at $x_{0}$ by assumption (1.1), $p$ must be continuous at 0 . From

we get

$$
p\left(h_{0}+h\right) \leq p\left(h_{0}\right)+p(h) \quad \text { and } \quad p\left(h_{0}\right) \leq p\left(h_{0}+h\right)+p(-h)
$$

$$
-p(-h) \leq p\left(h_{0}+h\right)-p\left(h_{0}\right) \leq p(h),
$$

that is, $p$ is continuous at $h_{0}$ as well. But then $p$ has a subgradient at $h_{0}$, that is, for some $x_{0}{ }^{\prime} \in X^{\prime}$

$$
\left\langle h-h_{0}, x_{0}{ }^{\prime}\right\rangle \leq p(h)-p\left(h_{0}\right) \quad \text { for all } h \in X .
$$

$h=0$ and $h=2 h_{0}$ show

$$
\left\langle-h_{0}, x_{0}{ }^{\prime}\right\rangle \leq-p\left(h_{0}\right), \quad\left\langle h_{0}, x_{0}{ }^{\prime}\right\rangle \leq p\left(2 h_{0}\right)-p\left(h_{0}\right)=p\left(h_{0}\right),
$$


and thus $\left\langle h_{0}, x_{0}{ }^{\prime}\right\rangle=p\left(h_{0}\right)=\left(y^{\prime} \circ f\right)^{\prime}\left(x_{0} ; h_{0}\right)$ and

$$
\left\langle h, x_{0}{ }^{\prime}\right\rangle \leq p(h)=\left(y^{\prime} \circ f\right)^{\prime}\left(x_{0} ; h\right) \quad \text { for all } h \in X .
$$

From proposition 2.1 we get $x_{0}{ }^{\prime} \in \partial\left(y^{\prime} \circ f\right)\left(x_{0}\right)$.

Because of proposition 2.1 the hyperplane

$$
\left\{x^{\prime} \in X^{\prime}:\left\langle h_{0}, x^{\prime}\right\rangle=\left\langle h_{0}, x_{0}{ }^{\prime}\right\rangle\right\}
$$

supports $\partial\left(y^{\prime} \circ f\right)\left(x_{0}\right)$ at $x_{0}{ }^{\prime}$ and consequently $x_{0}{ }^{\prime}$ is a boundary point of $\partial\left(y^{\prime} \circ f\right)\left(x_{0}\right)$. It will be a crucial fact for our construction below that under some assumptions on $X$ there exist boundary points $x^{\prime}$ of $\partial\left(y^{\prime} \circ f\right)\left(x_{0}\right)$ and supporting hyperplanes $H$ with $H \cap \partial\left(y^{\prime} \circ f\right)\left(x_{0}\right)=\left\{x^{\prime}\right\}$. To this let us call a point $x_{0}$ of a convex set $A$ in a locally convex vector space $E$ an exposed point of $A$, if there exists $l \in E^{\prime}$ such that $l\left(x_{0}\right)>l(x)$ for all $x \in A, x \neq x_{0}$ (see [2]). For later use we note a strengthening of the Krein-MilmanTheorem [2]:

Lemma 2.3. If $A$ is a convex weakly compact subset of a Banach space $E$ and $E$ is either separable or uniformly convex, then $A$ is the closure of the convex hull of the exposed points of $A$

$$
A=\operatorname{clconv} \exp A \text {. }
$$

In the following we denote by $\exp \partial\left(y^{\prime} \circ f\right)\left(x_{0}\right), y^{\prime} \in C^{\prime}$, the set of exposed points of $\partial\left(y^{\prime} \circ f\right)\left(x_{0}\right)$ where $X^{\prime}$ is endowed with any topology $\mathscr{T}$ consistent with $\left\langle X^{\prime}, X\right\rangle$ (that is, $\left.\sigma\left(X^{\prime}, X\right) \leq \mathscr{T} \leq \tau\left(X^{\prime}, X\right)\right)$. Since $\left({X^{\prime}}_{\sigma}\right)^{\prime}=$ $\left(X_{\tau}^{\prime}\right)^{\prime}$, this set is well-defined.

If $x_{0}{ }^{\prime} \in \exp \partial\left(y^{\prime} \circ f\right)\left(x_{0}\right)$ then for some $h_{0} \in X$

$$
\left\langle h_{0}, x_{0}{ }^{\prime}\right\rangle>\left\langle h_{0}, x^{\prime}\right\rangle \quad \text { for all } x^{\prime} \in \partial\left(y^{\prime} \circ f\right)\left(x_{0}\right), x^{\prime} \neq x_{0}{ }^{\prime},
$$

and from proposition 2.1 and 2.2 we obtain $\left\langle h_{0}, x_{0}{ }^{\prime}\right\rangle=\left(y^{\prime} \circ f\right)^{\prime}\left(x_{0} ; h_{0}\right)$, that is,

Proposition 2.4. Let $y^{\prime} \in C^{\prime}$ and $x_{0}{ }^{\prime} \in \exp \partial\left(y^{\prime} \circ f\right)\left(x_{0}\right)$. Then there exists $h_{0} \in X$ such that

$$
\left\langle h_{0}, x^{\prime}\right\rangle\left\langle\left\langle h_{0}, x_{0}{ }^{\prime}\right\rangle=\left(y^{\prime} \circ f\right)^{\prime}\left(x_{0} ; h_{0}\right) \quad \text { for all } x^{\prime} \in \partial\left(y^{\prime} \circ f\right)\left(x_{0}\right), x^{\prime} \neq x_{0}{ }^{\prime} .\right.
$$

We are now prepared to prove our main auxiliary result:

Proposition 2.5. Suppose $y_{0}{ }^{\prime} \in\left(C^{\prime}\right)^{\circ}$ and $x_{0}{ }^{\prime} \in \operatorname{convexp} \partial\left(y_{0}^{\prime} \circ f\right)\left(x_{0}\right)$. Then there exists a $S \in \mathscr{L}\left(Y^{\prime}{ }^{\prime}, X^{\prime}{ }_{\sigma}\right)$ such that $S y^{\prime} \in \partial\left(y^{\prime} \circ f\right)\left(x_{0}\right)$ for all $y^{\prime} \in C^{\prime}$. Moreover, $S y_{0}{ }^{\prime}=x_{0}{ }^{\prime}$. 
Proof. It is easily seen that it is sufficient to prove the assertion for $x_{0}{ }^{\prime} \in \exp \partial\left(y_{0}{ }^{\prime} \circ f\right)\left(x_{0}\right)$. Let $x_{0}{ }^{\prime}$ be such an element. To $y_{0}{ }^{\prime}$ and $x_{0}{ }^{\prime}$ fix $h_{0}$ as in proposition 2.4 and then choose for every $y^{\prime} \in C^{\prime}$ an element $x^{\prime}$ in $X^{\prime}$, say $S y^{\prime}$, such that

$$
S y^{\prime} \in \partial\left(y^{\prime} \circ f\right)\left(x_{0}\right), \quad\left\langle h_{0}, S y^{\prime}\right\rangle=\left(y^{\prime} \circ f\right)^{\prime}\left(x_{0} ; h_{0}\right) .
$$

That this can be done is the contents of proposition 2.2. Now let

$$
y^{\prime}=\sum_{i=1}^{k} \lambda_{i} y_{i}{ }^{\prime}, \quad y_{i}{ }^{\prime} \in C^{\prime}, \lambda_{i} \geq 0 \text { and } k \geq 1 .
$$

Going back to the definition of $\partial\left(y^{\prime} \circ f\right)\left(x_{0}\right)$ and $\left(y^{\prime} \circ f\right)^{\prime}\left(x_{0} ; h_{0}\right)$, it is easily verified that (2.2) holds as well if we replace $S y^{\prime}$ by $\sum_{i=1}^{k} \lambda_{i} S y_{i}^{\prime}$. Consequently

$$
S\left(\sum_{i=1}^{k} \lambda_{i} y_{i}{ }^{\prime}\right)=\sum_{i=1}^{k} \lambda_{i} S y_{i}^{\prime} \quad \text { for all } y_{i}{ }^{\prime} \in C^{\prime}, \lambda_{i} \geq 0, k \geq 1,
$$

if we assume that $S y^{\prime}$, for $y^{\prime} \in C^{\prime}$, is uniquely determined by (2.2). Because of proposition 2.4 this is true for $y_{0}{ }^{\prime}$, so that $S y_{0}{ }^{\prime}=x_{0}{ }^{\prime}$. Now suppose that for some $y_{1}{ }^{\prime} \in C^{\prime},(2.2)$ is satisfied by $x_{1}{ }^{\prime}, x_{1}{ }^{\prime \prime}, x_{1}{ }^{\prime} \neq x_{1}{ }^{\prime \prime}$. Since $y_{0}{ }^{\prime} \in\left(C^{\prime}\right)^{\circ}{ }_{\tau}$ we can choose $0<\lambda<1$ small enough such that

$$
y_{2}^{\prime}:=\frac{1}{1-\lambda} y_{0}^{\prime}-\frac{\lambda}{1-\lambda} y_{1}^{\prime} \in C^{\prime} ;
$$

thus $S y_{2}^{\prime}$ is defined. But then (2.2) holds for $y_{0}{ }^{\prime}=\lambda y_{1}{ }^{\prime}+(1-\lambda) y_{2}^{\prime}$, if we replace $S y_{0}^{\prime}$ by

$$
x^{\prime}:=\lambda x_{1}{ }^{\prime}+(1-\lambda) S y_{2}{ }^{\prime} \quad \text { or } \quad x^{\prime \prime}:=\lambda x_{1}^{\prime \prime}+(1-\lambda) S y_{2}{ }^{\prime},
$$

and thus $S y_{0}{ }^{\prime}=x^{\prime}=x^{\prime \prime}$ in contradiction to our assumption $x_{1}{ }^{\prime} \neq x_{1}{ }^{\prime \prime}$. So we see that $S$ maps $C^{\prime}$ "linearly" in the sense of $(2.3)$ into $X^{\prime}$. Since $\left(C^{\prime}\right)^{\circ}{ }_{\tau} \neq \varnothing$, every $y^{\prime} \in Y^{\prime}$ is representable in the form $y^{\prime}=y_{1}{ }^{\prime}-y_{2}{ }^{\prime}$ where $y_{1}{ }^{\prime}, y_{2}{ }^{\prime} \in C^{\prime}$. If $y^{\prime}=y_{3}{ }^{\prime}-y_{4}{ }^{\prime}$ is another representation of $y^{\prime}$ with $y_{3}{ }^{\prime}, y_{4}{ }^{\prime} \in C^{\prime}$, then $y_{1}{ }^{\prime}+y_{4}{ }^{\prime}=y_{3}{ }^{\prime}+y_{2}{ }^{\prime} \in C^{\prime}$ and from (2.3) we obtain

Thus by

$$
S y_{1}^{\prime}-S y_{2}^{\prime}=S y_{3}{ }^{\prime}-S y_{4}{ }^{\prime} \text {. }
$$

$$
S y^{\prime}:=S y_{1}{ }^{\prime}-S y_{2}{ }^{\prime} \quad \text { where } y^{\prime}=y_{1}^{\prime}-y_{2}{ }^{\prime}, \quad y_{1}{ }^{\prime}, y_{2}{ }^{\prime} \in C^{\prime},
$$

$S$ is uniquely extended to all of $Y$. Of course, $S$ is linear, $S y^{\prime} \in \partial\left(y^{\prime} \circ f\right)\left(x_{0}\right)$ for $y^{\prime} \in C^{\prime}$ and $S y_{0}{ }^{\prime}=x_{0}{ }^{\prime}$.

It remains to prove the continuity of $S$. To this end, let

$$
U:=\left\{x^{\prime} \in X^{\prime}:\left|\left\langle h_{1}, x^{\prime}\right\rangle\right| \leq 1\right\}, \quad h_{1} \in X,
$$

be given. We choose $\lambda>0$ small enough such that $x_{0} \pm \lambda h_{1} \in K$ and define $V \in N \tau\left(Y^{\prime}, Y\right)$ by 
where

$$
V:=\left\{y^{\prime} \in Y^{\prime}:\left|\left\langle y_{i}, y^{\prime}\right\rangle\right| \leq \lambda, i=1,2\right\}
$$

$$
y_{1}:=f\left(x_{0}+\lambda h_{1}\right)-f\left(x_{0}\right), \quad y_{2}:=f\left(x_{0}\right)-f\left(x_{0}-\lambda h_{1}\right) .
$$

For $y^{\prime} \in V \cap C^{\prime}$ we obtain from proposition 2.1, (2.1) and the definition of $V$

$$
\left\langle\lambda h_{1}, S y^{\prime}\right\rangle \leq\left(y^{\prime} \circ f\right)^{\prime}\left(x_{0} ; \lambda h_{1}\right) \leq\left\langle y_{1}, y^{\prime}\right\rangle \leq \lambda
$$

and similarly $\left\langle-\lambda h_{1}, S y^{\prime}\right\rangle \leq\left\langle-y_{2}, y^{\prime}\right\rangle \leq \lambda$, that is,

$$
S\left(V \cap C^{\prime}\right) \subset\left\{x^{\prime} \in X^{\prime}:\left|\left\langle\lambda h_{1}, x^{\prime}\right\rangle\right| \leq \lambda\right\}=U .
$$

Since $\left(C^{\prime}\right)^{\circ}{ }_{\tau} \neq \varnothing$ there exists a convex symmetric $W \in N \tau\left(Y^{\prime}, Y\right)$ and a $y^{\prime} \in C^{\prime}$ such that $y^{\prime}+W \subset C^{\prime}, y^{\prime}+W \subset V$ and thus

We get

$$
2 W=W-W=\left(y^{\prime}+W\right)-\left(y^{\prime}+W\right) \subset V \cap C^{\prime}-V \cap C^{\prime} .
$$

$$
S(2 W) \subset S\left(V \cap C^{\prime}-V \cap C^{\prime}\right) \subset U-U=2 U .
$$

This completes the proof.

Taking the adjoint of the above $S$ we obtain

Proposition 2.6. Suppose $X$ is a Mackey space (that is, $X$ has the topology $\left.\tau\left(X, X^{\prime}\right)\right), y_{0}{ }^{\prime} \in\left(C^{\prime}\right)^{\circ}{ }_{\tau}$ and $x_{0}{ }^{\prime} \in \operatorname{convexp} \partial\left(y_{0}{ }^{\prime} \circ f\right)\left(x_{0}\right)$. Then there exists a $T \in \partial f\left(x_{0}\right)$ such that $y_{0}{ }^{\prime} \circ T=x_{0}{ }^{\prime}$.

Proof. Let $S$ be the above constructed mapping and define for every $x \in X$ a linear form $T x$ on $Y^{\prime}$ by

$$
\left\langle T x, y^{\prime}\right\rangle:=\left\langle x, S y^{\prime}\right\rangle \quad \text { for all } y^{\prime} \in Y^{\prime} \text {. }
$$

Since $S \in \mathscr{L}\left(Y^{\prime}{ }^{\prime}, X^{\prime}{ }_{\sigma}\right)$ we have $T x \in\left(Y^{\prime}{ }_{\tau}\right)^{\prime}=Y$ for $x \in X$; but then $T \in \mathscr{L}\left(X_{\sigma}, Y_{\sigma}\right)$ and furthermore $T \in \mathscr{L}(X, Y)$, since $X$ is a Mackey space (see [6, chapter IV, 7.4]). By construction

$$
\left(y_{0}{ }^{\prime} \circ T\right)(x)=\left\langle T x, y_{0}{ }^{\prime}\right\rangle=\left\langle x, S y_{0}{ }^{\prime}\right\rangle=\left\langle x, x_{0}{ }^{\prime}\right\rangle
$$

for all $x \in X$, that is, $y_{0}{ }^{\prime} \circ T=x_{0}{ }^{\prime}$.

Now assume that $T \notin \partial f\left(x_{0}\right)$, hence $T\left(\bar{x}-x_{0}\right) \$ f(\bar{x})-f\left(x_{0}\right)$ for some $\bar{x} \in K$. Then the compact convex set $\{z\}$,

$$
z:=f(\bar{x})-f\left(x_{0}\right)-T\left(\bar{x}-x_{0}\right),
$$

and the closed convex set $C$ can be strictly separated by a closed hyperplane, that is, for some $y^{\prime} \in Y^{\prime}$ and $\lambda \in R$

$$
\left\langle y, y^{\prime}\right\rangle>\lambda>\left\langle z, y^{\prime}\right\rangle \quad \text { for all } y \in C \text {. }
$$


Since $C$ is a cone, we see that $y^{\prime} \in C^{\prime}, \lambda<0$ and thus $\left\langle z, y^{\prime}\right\rangle<0$, that is

$$
\left\langle\bar{x}-x_{0}, S y^{\prime}\right\rangle=\left\langle T\left(\bar{x}-x_{0}\right), y^{\prime}\right\rangle>y^{\prime} \circ f(\bar{x})-y^{\prime} \circ f\left(x_{0}\right)
$$

in contradiction to $S y^{\prime} \in \partial\left(y^{\prime} \circ f\right)\left(x_{0}\right)$.

\section{Main theorems.}

In order to be able to apply proposition 2.6 we have to make two assumptions: $\left(C^{\prime}\right)^{\circ}{ }_{r} \neq \varnothing$ and $\exp \partial\left(y^{\prime} \circ f\right)\left(x_{0}\right) \neq \varnothing$ for some $y^{\prime} \in\left(C^{\prime}\right)^{\circ}{ }_{r}$. Before we give a condition guaranteeing the existence of exposed points, let us note a simple consequence of $\left(C^{\prime}\right)^{\circ}{ }_{\tau} \neq \varnothing$. To this end, remember that the order cone $C$ is called normal with respect to a topology $\mathscr{T}$ on $Y$, if there exists a base of neighbourhoods $V$ of the origin in $\mathscr{T}$ such that

We have

$$
[u, z]:=\{y \in Y: u \leqq y \leqq z\} \subset V \text { if } u, z \in V .
$$

Proposition 3.1. If $\left(C^{\prime}\right)^{\circ} \neq \varnothing$ then $C$ is normal in $Y_{\sigma}$.

Proof. Since $\left(C^{\prime}\right)^{\circ} \neq \varnothing$ each $y_{0}{ }^{\prime} \in Y^{\prime}$ is representable in the form $y_{0}^{\prime}=y_{1}^{\prime}-y_{2}^{\prime}, y_{1}^{\prime}, y_{2}^{\prime} \in C^{\prime}$. Now

$$
\left\{y \in Y:\left|\left\langle y, y_{i}{ }^{\prime}\right\rangle\right| \leq 1, i=1,2\right\} \subset\left\{y \in Y:\left|\left\langle y, y_{0}{ }^{\prime}\right\rangle\right| \leq 2\right\} ;
$$

consequently the sets

$$
\left\{y \in Y:\left|\left\langle y, y_{i}{ }^{\prime}\right\rangle\right| \leq 1, i=1,2, \ldots, m\right\}, \quad y_{i}{ }^{\prime} \in C^{\prime},
$$

form a base for $N \sigma\left(Y, Y^{\prime}\right)$. But then the assertion follows easily from the fact that for $y_{i}^{\prime} \in C^{\prime}$ and $u \leqq y \leqq z$

$$
\left\langle u, y_{i}{ }^{\prime}\right\rangle \leq\left\langle y, y_{i}{ }^{\prime}\right\rangle \leq\left\langle z, y_{i}{ }^{\prime}\right\rangle \text {. }
$$

Now let $X$ be semireflexive and normable (hence a reflexive Banach space) and either separable or smoothly convex. Then $X^{\prime}{ }_{\tau}$ is a Banach space as well and furthermore separable respectively uniformly convex (see $[3, \S 26,10,(12)])$. From (1.4) it follows that $\partial\left(y^{\prime} \circ f\right)\left(x_{0}\right), y^{\prime} \in C^{\prime}$, is a nonvoid convex $\sigma\left(X^{\prime}, X^{\prime \prime}\right)$-compact subset of $X^{\prime}{ }_{\tau}$; hence by lemma 2.3

LEMMA 3.2. If $X$ is a reflexive Banach space and either separable or smoothly convex, then

Moreover

$$
\exp \partial\left(y^{\prime} \circ f\right)\left(x_{0}\right) \neq \varnothing \quad \text { for all } y^{\prime} \in C^{\prime} .
$$

$$
\partial\left(y^{\prime} \circ f\right)\left(x_{0}\right)=\operatorname{clconvexp} \partial\left(y^{\prime} \circ f\right)\left(x_{0}\right) \text {. }
$$

(Here the closure can be taken in any topology consistent with $\left\langle X^{\prime}, X\right\rangle$.) 
ReMaRK. Lemma 3.2 applies for instance to $X=l^{p}$ and $X=L^{p}$, $1<p<\infty$.

Now we can state our first theorem. The central result will be that $\partial f\left(x_{0}\right)$ is nonvoid; the other points are proved similarly as in [8].

\section{Theorem 3.3. If}

(a) $X$ is a reflexive Banach space and is either separable or smoothly convex, (b) $\left(C^{\prime}\right)^{\circ}{ }_{\tau} \neq \varnothing$,

then $\partial f\left(x_{0}\right)$ is a nonvoid convex equicontinuous subset of $\mathscr{L}\left(X, Y_{\sigma}\right)$.

If, furthermore,

(c) all order intervals $[u, z]$ are relatively compact in $\boldsymbol{Y}_{\sigma}$,

then $\partial f\left(x_{0}\right)$ is compact in $\mathscr{L}_{s}\left(X, Y_{\sigma}\right)$.

(Here $\mathscr{L}_{s}\left(X, Y_{\sigma}\right)$ is the space $\mathscr{L}\left(X, Y_{\sigma}\right)$ endowed with the topology of simple convergence.)

Proof. $\partial f\left(x_{0}\right) \neq \varnothing$ is an immediate consequence of assumptions (a), (b), lemma 3.2 and proposition 2.6. The convexity of $\partial f\left(x_{0}\right)$ is obvious.

Now let $V \in N \sigma\left(Y, Y^{\prime}\right)$ be given; as shown in the proof of proposition 3.1, we may assume that $V$ is symmetric and that $[u, z] \subset V$ if $u, z \in V$. Because of the continuity of $f$ in $x_{0}$ there exists a symmetric neighbourhood $U$ of 0 in $X$ such that $f\left(x_{0}+U\right)-f\left(x_{0}\right) \subset V$, that is,

$$
f\left(x_{0}+h\right)-f\left(x_{0}\right) \in V, \quad f\left(x_{0}\right)-f\left(x_{0}-h\right) \in-V=V
$$

for all $h \in U$. From $T h \leqq f\left(x_{0}+h\right)-f\left(x_{0}\right)$ and $T(-h) \leqq f\left(x_{0}-h\right)-f\left(x_{0}\right)$ for all $T \in \partial f\left(x_{0}\right)$ and $h \in U$ we get

$$
T h \in\left[f\left(x_{0}\right)-f\left(x_{0}-h\right), f\left(x_{0}+h\right)-f\left(x_{0}\right)\right] \subset V,
$$

showing that $\partial f\left(x_{0}\right)$ is an equicontinuous subset of $\mathscr{L}\left(X, Y_{\sigma}\right)$.

As we have seen, for each $h \in U$ the set $\left\{T h: T \in \partial f\left(x_{0}\right)\right\}$ is contained in some order interval and by (c) in a relatively compact subset of $Y_{\sigma}$. Since $U$ is absorbing this holds for all $h \in X$; hence $\partial f\left(x_{0}\right)$ is relatively compact in $\mathscr{L}_{s}\left(X, Y_{\sigma}\right)$ (by [1, chapitre $\left.3, \S 3, \mathrm{n}^{\circ} 5\right]$ ). The proof will be finished if we can show that $\partial f\left(x_{0}\right)$ is closed in $\mathscr{L}_{s}\left(X, Y_{\sigma}\right)$. To see this, note that $\mathscr{L}(X, Y)=\mathscr{L}\left(X, Y_{\sigma}\right)$. In fact $\mathscr{L}(X, Y) \subset \mathscr{L}\left(X, Y_{\sigma}\right)$ is trivial. Now for $T \in \mathscr{L}\left(X, Y_{\sigma}\right)$ and $y^{\prime} \in Y^{\prime}$, the mapping $x \rightarrow\left\langle T x, y^{\prime}\right\rangle$ is a continuous hence weakly continuous linear form on $X$, which is equivalent 
to $T \in \mathscr{L}\left(X_{\sigma}, Y_{\sigma}\right)$, and, since $X$ is a Mackey space, $T \in \mathscr{L}(X, Y)$ (by [6, chapter IV, 7.4]). Thus

$$
\begin{aligned}
\partial f\left(x_{0}\right) & =\bigcap_{x \in K}\left\{T \in \mathscr{L}(X, Y): T\left(x-x_{0}\right) \leqq f(x)-f\left(x_{0}\right)\right\} \\
& =\bigcap_{x \in K}\left\{T \in \mathscr{L}\left(X, Y_{\sigma}\right): T\left(x-x_{0}\right) \in f(x)-f\left(x_{0}\right)-C\right\}
\end{aligned}
$$

and the theorem follows from the fact that for each $x \in X$, the mapping $T \rightarrow T\left(x-x_{0}\right)$ from $\mathscr{L}_{s}\left(X, Y_{\sigma}\right)$ into $Y_{\sigma}$ is continuous, and that $C$ is closed in $Y_{\sigma}$.

Remark. Assumption (a) was only needed to guarantee the existence of at least one exposed point in $\partial\left(y^{\prime} \circ f\right)\left(x_{0}\right)$ for some $y^{\prime} \in\left(C^{\prime}\right)^{\circ}{ }_{r}$. Our theorem holds, of course, with any hypothesis yielding the existence of such a point.

In section 4 we will give a condition for $Y$ and $C$ under which the assumptions (b) and (c) are satisfied.

The function $f$ is even regular subdifferentiable at $x_{0}$ in the following sense

Theorem 3.4. Under the assumption (a), (b), (c) of Theorem 3.3:

$$
y^{\prime} \circ \partial f\left(x_{0}\right)=\partial\left(y^{\prime} \circ f\right)\left(x_{0}\right) \quad \text { for all } y^{\prime} \in C^{\prime} \text {. }
$$

Proof. Note first that $y^{\prime} \circ \partial f\left(x_{0}\right) \subset \partial\left(y^{\prime} \circ f\right)\left(x_{0}\right)$. Thus lemma 3.2 implies

$$
y^{\prime} \circ \partial f\left(x_{0}\right) \subset \partial\left(y^{\prime} \circ f\right)\left(x_{0}\right)=\mathrm{clconvexp} \partial\left(y^{\prime} \circ f\right)\left(x_{0}\right) \quad \text { for } y^{\prime} \in C^{\prime} .
$$

From proposition 2.6 we get

$$
\text { convexp } \partial\left(y^{\prime} \circ f\right)\left(x_{0}\right) \subset y^{\prime} \circ \partial f\left(x_{0}\right) \quad \text { for } y^{\prime} \in\left(C^{\prime}\right)^{\circ}{ }_{\tau} .
$$

Now for each $y^{\prime} \in Y^{\prime}$, the mapping $T \rightarrow y^{\prime} \circ T$ maps $\mathscr{L}_{s}\left(X, Y_{\sigma}\right)$ continuously into $X^{\prime}{ }_{\sigma}$ and consequently $y^{\prime} \circ \partial f\left(x_{0}\right)$, for $y^{\prime} \in C^{\prime}$, is compact in $X^{\prime}{ }_{\sigma}$. The assertion follows for $y^{\prime} \in\left(C^{\prime}\right)^{\circ}{ }_{\tau}$ from the above inclusions. Now, suppose $x^{\prime} \in \partial\left(y^{\prime} \circ f\right)\left(x_{0}\right)$ but $x^{\prime} \notin y^{\prime} \circ \partial f\left(x_{0}\right)$ for some $y^{\prime} \in C^{\prime}, y^{\prime} \notin\left(C^{\prime}\right)^{\circ}{ }_{\tau}$. Then by a separation argument

$$
\left(x^{\prime}+U\right) \cap\left(y^{\prime} \circ \partial f\left(x_{0}\right)+U\right)=\varnothing
$$

for some $U \in N \sigma\left(X^{\prime}, X\right)$, say $U=\left\{u \in X^{\prime}:|\langle\bar{x}, u\rangle| \leq 1\right\}$. For a fixed $y_{1}{ }^{\prime} \in\left(C^{\prime}\right)^{\circ}{ }_{r}$ and $x_{1}{ }^{\prime} \in \partial\left(y_{1}{ }^{\prime} \circ f\right)\left(x_{0}\right)$ we consider the sequences

$$
y_{j}^{\prime}:=j^{-1} y_{1}^{\prime}+\left(1-j^{-1}\right) y^{\prime}, \quad x_{j}^{\prime}:=j^{-1} x_{1}{ }^{\prime}+\left(1-j^{-1}\right) x^{\prime}, \quad j=1,2, \ldots
$$

Then $x_{j}{ }^{\prime} \in x^{\prime}+U$ for $j$ larger than some $j_{0}$. Moreover, it is easily verified 
that $x_{j}{ }^{\prime} \in \partial\left(y_{j}{ }^{\prime} \circ f\right)\left(x_{0}\right)$ for all $j$, and, since $y_{j}{ }^{\prime} \in\left(C^{\prime}\right)^{\circ}{ }_{\tau}$, that $x_{j}{ }^{\prime} \in y_{j}{ }^{\prime} \circ \partial f\left(x_{0}\right)$. Hence

$$
x_{j}^{\prime} \in\left(x^{\prime}+U\right) \cap y_{j} \circ \partial f\left(x_{0}\right) \quad \text { for } j \geq j_{0} .
$$

Since $\partial f\left(x_{0}\right)$ is compact in $\mathscr{L}_{s}\left(X, Y_{\sigma}\right)$, there exists $\lambda>0$ such that $\left|\left\langle T \bar{x}, y_{1}^{\prime}-y^{\prime}\right\rangle\right| \leq \lambda$ for all $T \in \partial f\left(x_{0}\right)$; hence

$$
\left|\left\langle\bar{x},\left(y_{j}^{\prime}-y^{\prime}\right) \circ T\right\rangle\right|=j^{-1}\left|\left\langle T \bar{x}, y_{1}{ }^{\prime}-y^{\prime}\right\rangle\right| \leq 1
$$

for $j$ larger than some $j_{1}$, that is,

and because of (3.1),

$$
y_{j}^{\prime} \circ \partial f\left(x_{0}\right) \subset y^{\prime} \circ \partial f\left(x_{0}\right)+U,
$$

$$
\left(x^{\prime}+U\right) \cap y_{j}^{\prime} \circ \partial f\left(x_{0}\right)=\varnothing
$$

for $j \geq j_{1}$. This contradicts (3.2).

REMARK. The assumptions of the above theorems are, for example, satisfied in the special case $X=\mathrm{R}^{n}, Y=\mathrm{R}^{m}$ and $C$ any closed proper convex cone in $R^{m}$. Since $R^{m}$ is order complete if and only if the closed cone $C$ is generated by $m$ linearly independent elements, our theorems are in the finite dimensional case a direct generalization of the results given by Valadier.

In order to give an example, where theorems 3.3 and 3.4 do not hold, let $Y$ be an ordered vector space with topology $\mathscr{T}>\sigma\left(Y, Y^{\prime}\right)$. Define $X:=Y_{\sigma}$ and consider any $f \in \mathscr{L}\left(X, Y_{\sigma}\right)$ but $f \notin \mathscr{L}(X, Y)$ (for example $f(x):=x$ for $x \in X) . f$ is a convex mapping satisfying (1.1) for $x_{0}=0$. It is easily verified that $\partial f(0)=\varnothing$ but $\partial\left(y^{\prime} \circ f\right)(0) \neq \varnothing$.

\section{Cones with a compact base.}

We will give a condition for $Y$ and $C$, under which $\left(C^{\prime}\right)^{\circ}{ }_{r} \neq \varnothing$ and all order intervals are relatively compact in $Y_{\sigma}$. In order to do this remember that a nonempty convex subset $B$ of $C$ is called a base for $C$ if each $y \in C, y \neq 0$, has a unique representation $y=\lambda b$, where $b \in B, \lambda \in \mathrm{R}, \lambda>0$ (see [5, chapter I, §3]). If $\left(C^{\prime}\right)^{\circ}{ }_{z} \neq \varnothing$ and $y_{0}{ }^{\prime} \in\left(C^{\prime}\right)^{\circ}{ }_{r}$ then the closed convex set

is a base for $C$. Now

$$
B:=\left\{y \in C:\left\langle y, y_{0}{ }^{\prime}\right\rangle=1\right\}
$$

$\left|\left\langle y, y^{\prime}\right\rangle\right| \leq 1$ for $y \in B$ and $y^{\prime} \in U:=\left(-y_{0}{ }^{\prime}+C^{\prime}\right) \cap\left(y_{0}^{\prime}-C^{\prime}\right)$,

and, since $U \in N \tau\left(Y^{\prime}, Y\right)$, the base $B$ is an equicontinuous subset of $\left({Y^{\prime}}^{\prime}\right)^{\prime}=Y$. By the theorem of Alaoglu-Bourbaki: 
Proposition 4.1. Suppose $\left(C^{\prime}\right)^{\circ}{ }_{\tau} \neq \varnothing$ and $y_{0}{ }^{\prime} \in\left(C^{\prime}\right)^{\circ}{ }_{\tau}$. Then

$$
B=\left\{y \in C:\left\langle y, y_{0}{ }^{\prime}\right\rangle=1\right\}
$$

is a weakly compact base for $C$.

If $Y$ is semireflexive then the converse of 4.1 holds:

Proposition 4.2. Suppose $Y$ is semireflexive and $C$ has a weakly compact base $B$ lying in a closed hyperplane $H$ not containing 0 . Then $\left(C^{\prime}\right)^{\circ}{ }_{\tau} \neq \varnothing$. Furthermore, all order intervals are relatively compact in $Y_{\sigma}$.

Proof. Let $B \subset H=\left\{y \in Y:\left\langle y, y_{0}{ }^{\prime}\right\rangle=1\right\}$ for some $y_{0}{ }^{\prime} \in Y^{\prime}$. Since $B$ is a base for $C$ we see that $y_{0}{ }^{\prime} \in C^{\prime}$. We will show that

$$
\left[-y_{0}{ }^{\prime}, y_{0}{ }^{\prime}\right]=\left(-y_{0}{ }^{\prime}+C^{\prime}\right) \cap\left(y_{0}{ }^{\prime}-C^{\prime}\right)
$$

is a barrel in $Y^{\prime}{ }^{\prime}$ (that is, a convex circled closed and absorbing set) and thus an element of $N \tau\left(Y^{\prime}, Y\right)$, since $Y^{\prime}{ }_{\tau}$ is barreled (by [6, chapter IV, 5.5]). But then $\left(C^{\prime}\right)^{\circ}{ }_{\tau} \neq \varnothing$, since

$$
y_{0}{ }^{\prime}+\left[-y_{0}{ }^{\prime}, y_{0}{ }^{\prime}\right] \subset C^{\prime} .
$$

Moreover, we see that $C$ is normal in $Y_{\sigma}$ (by proposition 3.1) and thus all order intervals are bounded in $Y_{\sigma}$, hence relatively compact in $Y_{\sigma}$ (by [6, chapter IV, 5.5]).

It is easily verified that $\left[-y_{0}{ }^{\prime}, y_{0}{ }^{\prime}\right]$ is convex, closed and circled since $y_{0}{ }^{\prime} \in C^{\prime}$. In order to see that $\left[-y_{0}{ }^{\prime}, y_{0}{ }^{\prime}\right]$ is absorbing let $y_{1}{ }^{\prime} \in Y^{\prime}$ be given. We will show that $y_{1}{ }^{\prime} \in \lambda\left[-y_{0}{ }^{\prime}, y_{0}{ }^{\prime}\right]$ where $\lambda>0$ is such that $B \subset \lambda U$ for

$$
U:=\left\{y \in Y:\left|\left\langle y, y_{1}{ }^{\prime}\right\rangle\right| \leq 1\right\}
$$

since $B$ is compact in $Y_{\sigma}$ such a $\lambda$ exists. Now, assume $y_{1}{ }^{\prime} \notin \lambda\left[-y_{0}{ }^{\prime}, y_{0}{ }^{\prime}\right]$, that is,

$$
\lambda^{-1} y_{1}^{\prime} \notin y_{0}^{\prime}-C^{\prime} \quad \text { or } \quad \lambda^{-1} y_{1}^{\prime} \notin-y_{0}^{\prime}+C^{\prime} .
$$

Let us consider only the case $\lambda^{-1} y_{1}{ }^{\prime} \notin y_{0}{ }^{\prime}-C^{\prime}$ (the other assumption can be dealt with similarly), that is,

$$
z^{\prime}:=y_{0}{ }^{\prime}-\lambda-1 y_{1}^{\prime} \notin C^{\prime} .
$$

By a separation argument there is a $y \in Y, y \neq 0$, and an $\alpha \in \mathrm{R}$ such that

$$
\left.\left\langle y, y^{\prime}\right\rangle>\alpha\right\rangle\left\langle y, z^{\prime}\right\rangle \quad \text { for all } y^{\prime} \in C^{\prime} .
$$

Since $C$ is a cone, we get $\alpha<0, y \in C^{\prime \prime}:=\left\{u \in Y:\left\langle u, C^{\prime}\right\rangle \geq 0\right\}$ and, by the bipolar theorem, $y \in C$, that is, $y=\beta b$ where $b \in B, \beta>0$. Thus 


$$
\beta^{-1}\left\langle y, z^{\prime}\right\rangle=\left\langle b, z^{\prime}\right\rangle=\left\langle b, y_{0}{ }^{\prime}\right\rangle-\lambda^{-1}\left\langle b, y_{1}{ }^{\prime}\right\rangle\left\langle\alpha \beta^{-1}<0,\right.
$$

hence $\lambda^{-1}\left\langle b, y_{1}{ }^{\prime}\right\rangle>\left\langle b, y_{0}{ }^{\prime}\right\rangle=1$, that is, $b \notin \lambda U$ in contradiction to the choice of $\lambda$.

From proposition 4.2. we get

Theorem 4.3. Let $Y$ be semireflexive. Then theorem 3.3 and 3.4 hold if the assumptions (b) and (c) are replaced by

(b') $C$ has a weakly compact base lying in a closed hyperplane not running through 0.

REMark. It is easy to construct closed proper convex cones $C$ satisfying $\left(b^{\prime}\right)$. For this purpose let $H$ be a closed hyperplane in $Y$ not containing 0 , take a nonempty convex weakly compact subset $B$ in $H$ and define $C:=\bigcup_{\lambda \geq 0} \lambda B$.

\section{Subdifferentiability and Gateaux differentiability.}

Let us note an interesting conclusion from proposition 4.1. Recall that the infimum (if it exists) of the set

$$
\left\{\lambda^{-1}\left(f\left(x_{0}+\lambda h\right)-f\left(x_{0}\right)\right): \lambda>0, x_{0}+\lambda h \in K\right\}
$$

is called the directional derivative $f^{\prime}\left(x_{0} ; h\right)$ of $f$ at $x_{0}$ in the direction $h$ (cf. [8]).

Theorem 5.1. If $\left(C^{\prime}\right)^{\circ} \neq \varnothing$ then $f^{\prime}\left(x_{0} ; h\right)$ is defined for all $h \in X$. If, in addition, $C$ is normal, then

$$
f^{\prime}\left(x_{0} ; h\right)=\lim \lambda^{-1}\left(f\left(x_{0}+\lambda h\right)-f\left(x_{0}\right)\right), \quad \lambda \rightarrow 0, \lambda>0 .
$$

Proof. Let $h \in X$ and assume $x_{0} \pm h \in K$ (otherwise replace $h$ by $\lambda h$, $\lambda>0$ sufficiently small). For $0<\mu \leq \nu \leq 1$ we have

$$
f\left(x_{0}+\mu h\right)=f\left(\frac{\nu-\mu}{\nu} x_{0}+\frac{\mu}{\nu}\left(x_{0}+\nu h\right)\right) \leqq \frac{\nu-\mu}{\nu} f\left(x_{0}\right)+\frac{\mu}{\nu} f\left(x_{0}+\nu h\right)
$$

and hereby

$$
\mu^{-1}\left(f\left(x_{0}+\mu h\right)-f\left(x_{0}\right)\right) \leqq \nu^{-1}\left(f\left(x_{0}+\nu h\right)-f\left(x_{0}\right)\right), \quad 0<\mu \leq \nu \leq 1 .
$$

Similarly one gets

$$
f\left(x_{0}\right)-f\left(x_{0}-h\right) \leqq \mu^{-1}\left(f\left(x_{0}+\mu h\right)-f\left(x_{0}\right)\right) \quad \text { for } 0<\mu \leq 1
$$

and thus with 


$$
y_{n}:=n\left(f\left(x_{0}+n^{-1} h\right)-f\left(x_{0}\right)\right)-f\left(x_{0}\right)+f\left(x_{0}-h\right), \quad n=1,2, \ldots,
$$

we have

$$
0 \leqq y_{n} \leqq y_{m} \quad \text { for } n \geq m, n, m \in \mathrm{N} .
$$

We will show that the sequence $\left\{y_{n}\right\}_{n \in N}$ converges in $Y_{\sigma}$ to some $y$. This yields for each $n_{0} \in \mathrm{N}$ that

$$
y \in \operatorname{cl}\left\{y_{n}: n \geq n_{0}\right\} \subset \operatorname{cl}\left(y_{n_{0}}-C\right)=y_{n_{0}}-C,
$$

that is, $y$ is a lower bound for $\left\{y_{n}\right\}_{n \in \mathbb{N}}$. If $z$ is any other lower bound, that is, $z \leqq y_{n}$ for all $n$, then $y_{n}-z \in C$ and thus $y-z \in \bar{C}=C$, hence $y=\inf \left\{y_{n}\right\}$. The first part of the assertion is an easy consequence from this.

As shown in section 4 each $y_{0}{ }^{\prime} \in\left(C^{\prime}\right)^{\circ}{ }_{\tau}$ determines a representation $y_{n}=\lambda_{n} b_{n}$ where

$$
b_{n} \in B:=\left\{y \in C:\left\langle y, y_{0}{ }^{\prime}\right\rangle=1\right\}
$$

and $\lambda_{n} \geq 0$. From $0 \leqq y_{n} \leqq y_{m}$ for $n \geq m$ we obtain $0 \leq \lambda_{n} \leq \lambda_{m}$, so that $\left\{\lambda_{n}\right\}$ converges. Moreover, for some $\lambda>0$ sufficiently large $\left\{y_{n}\right\} \subset \operatorname{conv}(0 \cup \lambda B)$. As conv $(0 \cup \lambda B)$ is a compact subset of $Y_{\sigma}$, the convergence of $\left\{y_{n}\right\}$ will follow, if we can show that $\left\{y_{n}\right\}$ is a Cauchy sequence in $Y_{\sigma}$. To see this, let $y^{\prime} \in Y^{\prime}$ be given. Then $y^{\prime}=y_{1}{ }^{\prime}-y_{2}{ }^{\prime}$ where $y_{1}{ }^{\prime}, y_{2}{ }^{\prime} \in\left(C^{\prime}\right)^{\circ}{ }_{r}$. If $y_{n}=$ $\alpha_{n} u_{n}=\beta_{n} v_{n}$ are the representations of $y_{n} \in\left\{y_{n}\right\}_{n \in \mathrm{N}}$ with respect to the bases given by $y_{1}{ }^{\prime}, y_{2}^{\prime}$, then

$$
\left|\left\langle y_{i}-y_{j}, y^{\prime}\right\rangle\right| \leq\left|\left\langle y_{i}-y_{j}, y_{1}{ }^{\prime}\right\rangle\right|+\left|\left\langle y_{i}-y_{j}, y_{2}{ }^{\prime}\right\rangle\right|=\left|\alpha_{i}-\alpha_{j}\right|+\left|\beta_{i}-\beta_{j}\right|
$$

and this converges to 0 , if $i, j \rightarrow \infty$. Thus $\left\{y_{n}\right\}$ is a Cauchy sequence in $Y_{\sigma}$.

Since $C$ is normal in $Y_{\sigma}$ (by proposition 3.1 ), it is an easy consequence of (5.1) that

$$
f^{\prime}\left(x_{0} ; h\right)=\lim \lambda^{-1}\left(f\left(x_{0}+\lambda h\right)-f\left(x_{0}\right)\right), \quad \lambda \rightarrow 0, \lambda>0,
$$

in $Y_{\sigma}$. If in addition $C$ is normal in $Y$, this even holds in $Y$ (by [5, chapter $2,3.4])$.

Theorem 5.1 shows that the above definition of the directional derivative is in accordance with the definition used in 2.

Recall that $f$ is called Gateaux differentiable at $x_{0}$, if

$$
\lim \lambda^{-1}\left(f\left(x_{0}+\lambda h\right)-f\left(x_{0}\right)\right), \quad \lambda \rightarrow 0,
$$

exists for all $h \in X([3, \S 26,4])$. Since for a convex function $f: \mathrm{R}^{n} \rightarrow \mathrm{R}$ the partial derivatives (if they exist) are continuous (see [7, Theorem 4.4.7]), the Gateaux- and Fréchet-differentiability coincide for such an $f$. Thus 5.2 is a generalization of the well-known theorem that a convex 
function $f: \mathrm{R}^{n} \rightarrow \mathrm{R}$ is differentiable at $x_{0}$ if and only if $f$ has a unique subgradient at $x_{0}$ (that is, there exists only one "nonvertical" supporting hyperplane at $\left(x_{0}, f\left(x_{0}\right)\right)$ to

$$
\left.\left\{(x, z) \in \mathrm{R}^{n+1}: f(x) \leq z\right\}\right)
$$

(cf. [7, Theorem 4.4.6]).

THEOREM 5.2. If assumptions (a), (b), (c) of theorem 3.3 hold and if $C$ is normal in $Y$, then $f$ is Gateaux differentiable at $x_{0}$ if and only if $f$ has a unique subgradient at $x_{0}$.

Proof. Suppose

$$
d\left(x_{0} ; h\right):=\lim \lambda^{-1}\left(f\left(x_{0}+\lambda h\right)-f\left(x_{0}\right)\right), \quad \lambda \rightarrow 0,
$$

exists for all $h \in X$. Let $T \in \partial f\left(x_{0}\right)$. Then for $h \in X$ and all $\lambda>0$ sufficiently small

$$
\lambda T h=T\left(x_{0}+\lambda h-x_{0}\right) \leqq f\left(x_{0}+\lambda h\right)-f\left(x_{0}\right),
$$

hence $T h \leqq d\left(x_{0} ; h\right)$. For $-h$ we get

$$
-T h=T(-h) \leqq d\left(x_{0} ;-h\right)=-d\left(x_{0} ; h\right)
$$

and thus $T h=d\left(x_{0} ; h\right)$ for all $h$, which shows that $T$ is uniquely determined.

Now, suppose that $f$ is not Gateaux differentiable at $x_{0}$, and let us show that $\partial f\left(x_{0}\right)$ contains at least two elements. From theorem 5.1 we get $f^{\prime}\left(x_{0} ; h\right) \neq-f^{\prime}\left(x_{0} ;-h\right)$ for some $h$, hence

that is,

$$
\left\langle f^{\prime}\left(x_{0} ; h\right), y^{\prime}\right\rangle \neq\left\langle-f^{\prime}\left(x_{0} ;-h\right), y^{\prime}\right\rangle \quad \text { for some } y^{\prime} \in C^{\prime},
$$

$$
\left(y^{\prime} \circ f\right)^{\prime}\left(x_{0} ; h\right) \neq-\left(y^{\prime} \circ f\right)^{\prime}\left(x_{0} ;-h\right) .
$$

We choose $x_{1}{ }^{\prime}, x_{2}{ }^{\prime} \in \partial\left(y^{\prime} \circ f\right)\left(x_{0}\right)$ as in proposition 2.2,

$$
\left\langle h, x_{1}{ }^{\prime}\right\rangle=\left(y^{\prime} \circ f\right)^{\prime}\left(x_{0} ; h\right) \text { and }\left\langle-h, x_{2}{ }^{\prime}\right\rangle=\left(y^{\prime} \circ f\right)^{\prime}\left(x_{0} ;-h\right) \text {, }
$$

and thus $\left\langle h, x_{1}{ }^{\prime}\right\rangle \neq\left\langle h, x_{2}{ }^{\prime}\right\rangle$, that is $x_{1}{ }^{\prime} \neq x_{2}{ }^{\prime}$. The remaining part of the proof follows from theorem 3.4.

Note ADDED IN PROOF. Recently M. M. Day pointed out that Lemma 2.3 holds without the assumption that $E$ is either separable or uniformly convex (M. M. Day, Normed linear spaces, 3. edition, Springer-Verlag, Heidelberg · New York, 1973, ch. III, 5, 5a). Consequently in Lemma 3.2 and Theorem 3.3 the hypothesis that $X$ is either separable or smoothly convex can be omitted. 


\section{REFERENCES}

1. N. Bourbaki, Espaces vectoriels topologiques, chapitres 3, 4, 5 (Act. Sci. Ind. 1229), lre éd., Hermann, Paris, 1966.

2. V. L. Klee, Extremal structure of convex sets II, Math. Z., 69 (1958), 90-104.

3. G. Köthe, Topologische lineare Räume I (Grundlehren Math. Wissensch. 107), SpringerVerlag, Berlin - Göttingen - Heidelberg, 1960.

4. J. J. Moreau, Fonctionelles convexes (Séminaire sur les équations aux dérivées partielles II), Collège de France, Paris, 1966-67.

5. A. L. Peressini, Ordered topological vector spaces, Harper and Row, New York, 1967.

6. H. H. Schaefer, Topological vector spaces, Macmillan, New York, 1966.

7. J. Stoer, C. W. Witzgall, Convexity and optimization in finite dimensions I (Grundlehren Math. Wissensch. 163), Springer-Verlag, Berlin · Heidelberg - New York, 1970.

8. M. Valadier, Sous-différentiabilité de fonctions convexes à valeurs dans un espace vectoriel ordonné, Math. Scand. 30 (1972), 65-74. 